\title{
NESTED SEQUENCES OF LOCAL UNIFORM SPACES
}

\author{
BY
}

\author{
JAMES WILLIAMS
}

\begin{abstract}
A locally uniform space is a pair of sets $(X, \mathscr{V}), \mathscr{V}$ being a filter on $X \times X$ such that $\forall U \in \mathscr{V}, \Delta(X)=\{\langle x, x\rangle: x \in X\} \subseteq U, U^{-1}=\{\langle y, x\rangle:\langle x, y\rangle \in U\}$ $\in \mathscr{V}$, and $\forall x \in X, \exists V \in \mathscr{V}:(V \circ V)[x] \subseteq U[x]$. We shall say that a sequence $\left\{\left(X_{n}, \mathscr{V}_{n}\right): n \in \omega\right\}$ is nested iff $\forall n \in \omega, X_{n} \subseteq X_{n+1}$ and $\mathscr{V}_{n+1} \mid X_{n}=\mathscr{V}_{n}$. By a limit for a nested sequence $\left\{\left(X_{n}, \mathscr{V}_{n}\right): n \in \omega\right\}$, we shall mean any locally uniform space $(X, \mathscr{V})$ such that $X=\bigcup\left\{X_{n}: n \in \omega\right\}$ and $\forall n \in \omega, \mathscr{V} \mid X_{n}=\mathscr{V}_{n}$. Our first task will be to consider when a nested sequence of locally uniform spaces has a limit; in order to do this, we shall introduce a weak generalization of pseudo-metric functions. We shall also show that, in contrast to locally uniform spaces, each nested sequence of uniform spaces has a limit.

With each locally uniform space one can associate a regular relative topology in the obvious fashion. E. Hewitt and J. Novak have constructed regular spaces of the type on which every real-valued continuous function is constant; we shall use our results about limits to give a relatively simple general construction for producing locally uniform spaces which have this type of relative topology. The construction may be done in such a way that the spaces produced have several pleasant topological properties.
\end{abstract}

1. Sequential functions. In this section we wish to study a class of spaces which does (in weaker fashion) for locally uniform spaces what pseudo-metric spaces (or more precisely, écart spaces) do for uniform spaces. For any set $X$, we shall let ${ }^{\omega>} X^{*}$ be the set of all finite sequences of elements of $X$. For each sequence $\sigma=x_{1}, \ldots, x_{n}$ in ${ }^{\omega>} X$ and each $x \in X$, let $\sigma x$ be the sequence $x_{1}, \ldots, x_{n}, x$.

Definition. A sequential function for a set $X$ is a function $S$ from ${ }^{\omega>} X$ to the power set of $X \times X$ such that $\forall \sigma \in{ }^{\omega>} X ;\{\langle x, x\rangle: x \in X\} \subseteq S_{\sigma}, S_{\sigma}$ is symmetric, and $\forall x \in X,\left(S_{\sigma x} \circ S_{\sigma x}\right)[x] \subseteq S_{\sigma}[x]$.

Definition. A gauge for a locally uniform space $(X, \mathscr{V})$ is a collection $\Gamma$ of sequential functions for $X$ such that $\bigcup\left\{S\left[{ }^{\omega>} X\right]: S \in \Gamma\right\}$ is a subbase for $\mathscr{V}$. We shall say that a locally uniform space is sequenceable iff it has a one element gauge.

1.1 THEOREM. Every locally uniform space has a gauge.

Proof. Let $(X, \mathscr{V})$ be a locally uniform space. For each symmetric $V$ of $\mathscr{V}$ we build a sequential function $S^{V}$ as follows: Let $S_{\phi}^{V}=V$; by induction, for each $\sigma \in{ }^{\omega>} X$ and each $\sigma \in X$, having chosen $S_{\sigma}^{V}$, we let $S_{\sigma x}^{V}$ be a symmetric element of $\mathscr{V}$ such that $\left(S_{\sigma x}^{V} \circ S_{\sigma x}^{V}\right)[x] \subseteq S^{V}[x]$. Then it is clear that $\left\{S^{V}: V\right.$ is a symmetric element of $\mathscr{V}\}$ is a gauge for $(X, \mathscr{V})$.

Received by the editors September 28, 1970 and, in revised form, September 8, 1971.

AMS 1969 subject classifications. Primary 5430, 5425, 5420.

Copyright (C) 1972, American Mathematical Society 
1.2 THeOREM. Any nonempty collection $\Gamma$ of sequential functions for a set $X$ is a gauge for some locally uniform space $(X, \mathscr{V})$.

Proof. Let $\Gamma$ be a nonempty collection of sequential functions for a set $X$. First, for each $S \in \Gamma, S\left[{ }^{\omega>} X\right]$ is a subbase for a local uniformity on $X$ by the definition of sequential functions. Second, it is easy to see that the union of any nonempty family of subbases for local uniformities on $X$ must itself be a subbase for a local uniformity on $X$. Hence $\bigcup\left\{S\left[{ }^{\omega>} X\right]: S \in \Gamma\right\}$ is a subbase for a local uniformity $\mathscr{V}$ on $X$, and $\Gamma$ is a gauge for $(X, \mathscr{V})$.

1.3 Proposition. A locally uniform space $(X, \mathscr{V})$ is sequenceable iff it has a subbase $\mathscr{B}$ with cardinality at most that of $X$.

Proof. If $(X, \mathscr{V})$ is finite, then it has a one element base $\{D\}$ which is generated by the sequential function $S$ given by $\forall \sigma \in{ }^{\omega>} X, S_{\sigma}=D$. We may thus assume that $X$ is infinite. If $S$ is a sequential function such that $S\left[{ }^{\omega>} X\right]$ is a subbase for $\mathscr{V}$, then $S\left[{ }^{\omega>} X\right]=\leqq X=. \aleph_{0}=X^{=}$. On the other hand, suppose $\mathscr{B}$ is a subbase for $\mathscr{V}$ and $\mathscr{B}=\leqq X=$ Let $A \subseteq X$ be such that $A=\mathscr{B}=$, and let $\left\{V_{x}: x \in A\right\}$ be an indexing of $\mathscr{B}$ by $A$. We may build a sequential function $S$ by requiring $S_{\phi}=X \times X$ and $\forall x \in A$, $S_{x}=V_{x} \cap V_{x}^{-1}$, and continue by induction to choose an appropriate $S_{\sigma} \in \mathscr{V}$ for each $\sigma \in{ }^{\omega} X$. Then $S\left[{ }^{\omega>} X\right] \supseteq \mathscr{B}$, so that $(X, \mathscr{V})$ is sequenceable.

1.4 Proposition. For each cardinal $\boldsymbol{N} \geqq \boldsymbol{\aleph}_{0}$, there is a nonsequenceable uniform space of cardinality $\aleph$.

Proof. Let $A$ be a set of cardinality $\boldsymbol{\aleph}$, and let $X=A \times \omega$, so that $X=\boldsymbol{\aleph}$. For each $f \in{ }^{A} \omega$ and each $x \in X$,

let $M_{f}(x)=\{\langle x, y\rangle: y \geqq f(x)\}$;

let $V_{f}=\Delta(X) \cup\left\{M_{f}(x) \times M_{f}(x): x \in X\right\}$.

Then $\left\{V_{f}: f \in{ }^{A} \omega\right\}$ is a base for a uniformity $\mathscr{V}$ of $X$ since, as is easy to verify, each $V_{f}$ is symmetric and contains the diagonal in $X \times X, V_{f} \circ V_{f}=V_{f}$, and $\forall g \in{ }^{A} \omega$, if $h \in{ }^{A} \omega$ is given by $h(x)=\max \{f(x), g(x)\}$, then $V_{h} \subseteq V_{f} \cap V_{g}$. Suppose $\mathscr{V}$ has a subbase of cardinality $\boldsymbol{\kappa}$; taking the set of all finite intersections of elements of such a subbase gives a base $\left\{U_{x}: x \in A\right\}$ of cardinality $\boldsymbol{\aleph}$, which we may assume to be indexed by $A$. For each $x \in A$, choose $f_{x} \in{ }^{A} \omega$ so that $V_{f_{x}} \subseteq U_{x}$. Then let $g \in{ }^{A} \omega$ be given by $g(x)=f_{x}(x)+1$. No $U_{x}$ is contained in $V_{g}$, since for each $x \in A$, $V_{g}[\langle x, g(x)\rangle]=M_{g}(x)$ is a proper subset of $M_{f_{x}}(x) \subseteq U_{x}[\langle x, f(x)\rangle]$. This contradicts the assumption that $\left\{U_{x}: x \in A\right\}$ is a base for $\mathscr{V}$.

2. Nested sequences of locally uniform spaces. For the following proposition we shall need the result, shown by J. R. Isbell [3], that a bounded uniformly continuous pseudo-metric on a subspace of a uniform space may be extended to a bounded uniformly continuous pseudo-metric on the whole space.

2.1 Proposition. Let $\left\{\left(X_{n}, \mathscr{U}_{n}\right): n \in \omega\right\}$ be a nested sequence of uniform spaces; let $X=\bigcup\left\{X_{n}: n \in \omega\right\}$. Then there is a strongest uniformity $\mathscr{U}$ on $X$ such that $\forall n \in \omega, \mathscr{U} \mid X_{n}=\mathscr{U}_{n}$. 
Proof. Let $\Gamma$ be the set of all pseudo-metrics $d$ on $X$ such that for each $n \in \omega$, $d \mid X_{n}$ is a bounded uniformly continuous pseudo-metric on $\left(X_{n}, \mathscr{U}_{n}\right)$. First we shall show for each $n \in \omega$, that each bounded uniformly continuous pseudo-metric $d$ on $\left(X_{n}, \mathscr{U}_{n}\right)$ is the restriction of an element of $\Gamma$. Using Isbell's result, we may find by induction, for each $k \geqq n$, a bounded uniformly continuous extension $d_{k+1}$ of $d_{k}$ to $\left(X_{k+1}, \mathscr{U}_{k+1}\right)$. If we let $d=\bigcup\left\{d_{k}: k \geqq n\right\}$, then $d$ is easily seen to be a pseudometric of the type belonging to $\Gamma$, and $d \mid X_{n}=d_{n}$. Let $\mathscr{I l}$ be the uniformity for which $\Gamma$ is a gauge. What we have just shown about $\Gamma$ assures $\mathscr{U} \mid X_{n} \supseteq \mathscr{U}_{n}$, and the definition of $\Gamma$ assures $\mathscr{U} \mid X_{n} \subseteq \mathscr{U}_{n} . \mathscr{U}$ is of course the strongest limit uniformity since for any limit uniformity $\mathscr{U}^{\prime}$, every bounded uniformly continuous pseudo-metric on $\left(X, \mathscr{U}^{\prime}\right)$ must belong to $\Gamma$.

Definition. A limit for a nested sequence $\left\{\left(X_{n}, \mathscr{V}_{n}\right): n \in \omega\right\}$ of locally uniform spaces is any locally uniform space $(X, \mathscr{V})$ such that $X=\bigcup\left\{X_{n}: n \in \omega\right\}$ and $\forall n \in \omega$, $\mathscr{V} \mid X_{n}=\mathscr{V}_{n}$. Such a limit is not in general unique.

2.2 THEOREM. A nested sequence of locally uniform spaces with completely regular topologies need not have a limit.

Proof by example. The proof depends entirely upon topological properties. Let $T$ be a completely regular space which is not normal. Let $A, B$ be closed disjoint subsets of $T$ which cannot be separated by open sets. Let $\omega$ be the natural numbers with the discrete topology. For each $j \in \omega$, let $X_{j}$ be the union of $\omega \times(A \cup B)$ and $\{k \in \omega: k<j\} \times T$, together with two points $a$ and $b$, which have, for each $k \in \omega$, the basic neighborhoods

$$
\begin{aligned}
& N_{k}(a)=\{a\} \cup\{\langle n, x\rangle: n>k, x \in A\}, \\
& N_{k}(b)=\{b\} \cup\{\langle n, y\rangle: n>k, y \in B\} .
\end{aligned}
$$

Then each $X_{j}$ is obviously completely regular, and a closed subspace of $X_{j+1}$. Let $X=\bigcup\left\{X_{j}: j \in \omega\right\}$; let $\mathscr{T}$ be any topology on $X$ such that $\forall j \in \omega, \mathscr{T} \mid X_{j}$ is the given topology on $X_{j}$. We shall show that $\mathscr{T}$ is not regular.

Let $M$ and $N$ be any open neighborhoods of $a$ and $b$ in $X$. Then we may choose $k_{0} \in \omega$ so that $\forall k>k_{0},\{k\} \times A \subseteq M$ and $\{k\} \times B \subseteq N$. For each $k>k_{0}, M$ contains a $\{k\} \times T$-neighborhood of $\{k\} \times A$, and hence $\bar{M}$ meets $\{k\} \times B \subseteq N$. Consequently $a$ and $b$ do not have disjoint closed neighborhoods. Since $a$ and $b$ have different neighborhood systems, $\mathscr{T}$ cannot be regular.

As for the part about local uniformities, we may just take each local uniformity $\mathscr{V}_{j}$ to be the set of all neighborhoods of the diagonal in $X_{j}$ (each $\mathscr{V}_{j}$ is in fact a local uniformity by Theorem 1.4 of [4]). We need to show that each $\left(X_{j}, \mathscr{V}_{j}\right)$ is a subspace of $\left(X_{j+1}, \mathscr{V}_{j+1}\right)$. Pick $V_{j} \in \mathscr{V}_{j}$; then $V_{j}$ is the restriction to $X_{j} \times X_{j}$ of an $X_{j+1} \times X_{j+1}$-neighborhood $W$ of $\Delta\left(X_{j}\right)$, the diagonal in $X_{j} \times X_{j}$. Let $V_{j+1}$ $=W \cup\left(\left(X_{j+1}-X_{j}\right) \times\left(X_{j+1}-X_{j}\right)\right)$; then $V_{j+1}$ is a neighborhood of the diagonal in $X_{j+1} \times X_{j+1}$ since $X_{j}$ is closed in $X_{j+1}$. Moreover $V_{j+1} \cap\left(X_{j} \times X_{j}\right)=V_{j}$ by choice of $W$. Hence $\mathscr{V}_{j+1} \mid X_{j}=\mathscr{V}_{j}$. We conclude that the nested sequence $\left\{\left(X_{j}, \mathscr{V}_{j}\right): j \in \omega\right\}$ fails to have a limit. 
Definition. If $(X, \mathscr{U})$ is a subspace of the locally uniform space $(Y, \mathscr{V})$ and $\Gamma$ is a gauge for $(Y, \mathscr{V})$, then we may restrict $\Gamma$ to a gauge for $(X, \mathscr{U})$ as follows: For each $S \in \Gamma$, we let the map $S \| X:{ }^{\omega>} X \rightarrow \mathscr{U}$ be given by $\forall \sigma \in{ }^{\omega>} X,(S \| X)_{\sigma}$ $=S_{\sigma} \cap(X \times X)$. It is clear that $S \| X$ is a sequential function, and if we let $\Gamma \mid X$ $=\{S \| X: S \in \Gamma\}$, then $\Gamma \mid X$ must be a gauge for $(X, \mathscr{U})$. (In the converse direction, however, if $\Gamma^{\prime}$ is a gauge for $(X, \mathscr{U})$, it may not be possible to find a gauge for $(Y, \mathscr{V})$ which restricts to $\Gamma^{\prime}$; this is evident by comparison of the following and preceding theorems.)

2.3 ThEOREM. Let $\left\{\left(X_{n}, \mathscr{V}_{n}\right): n \in \omega\right\}$ be a nested sequence of locally uniform spaces, then

I. There is a limit locally uniform space iff there is a collection of gauges $\Gamma_{n}$ for $\left(X_{n}, \mathscr{V}_{n}\right)$ such that $\forall n \in \omega, \Gamma_{n+1} \mid X_{n}=\Gamma_{n}$.

II. If there is a limit $\mathscr{V}$, then there is a strongest limit; there is also a limit which is strongest among all limits generating the same topology as $\mathscr{V}$.

Proof. To prove the first assertion, we first suppose $(X, \mathscr{V})$ is a limit of the sequence $\left\{\left(X_{n}, \mathscr{V}_{n}\right): n \in \omega\right\}$. Let $\Gamma$ be a gauge for $\mathscr{V}$; then $\forall n \in \omega, \Gamma \mid X_{n}$ is a gauge for $\left(X_{n}, \mathscr{V}_{n}\right)$ and $\left(\Gamma \mid X_{n+1}\right)\left|X_{n}=\Gamma\right| X_{n}$.

Now suppose that for each $n \in \omega, \Gamma_{n}$ is a gauge for $\left(X_{n}, \mathscr{V}_{n}\right)$ and $\Gamma_{n+1} \mid X_{n}=\Gamma_{n}$. Let $\Gamma$ be the set of all sequential functions $S$ for $X$ such that $\forall n \in \omega, S \| X_{n} \in \Gamma_{n}$. First we shall show for each $n \in \omega$ and each sequential function ${ }_{n} S \in \Gamma_{n}$, that there is a sequential function $S \in \Gamma$ such that $S \| X_{n}={ }_{n} S$. Using our assumption that for each $k, \Gamma_{k+1} \mid X_{k}=\Gamma_{k}$, we may choose by induction, for each $k \geqq n,{ }_{k+1} S \in \Gamma_{k+1}$ so that ${ }_{k+1} S \| X_{k}={ }_{k} S$. We now define a sequential function $S$ as follows: For each $\sigma \in{ }^{\omega>} X$, let $S_{\sigma}=\bigcup\left\{{ }_{k} S_{\sigma}: k \in \omega\right\}$. It is clear that each $S_{\sigma}$ is symmetric and contains the diagonal in $X \times X$. For each $\sigma \in{ }^{\omega>} X$ and each $x \in X$,

$$
\begin{aligned}
\left(S_{\sigma x} \circ S_{\sigma x}\right)[x] & =\left(\bigcup\left\{{ }_{p} S_{\sigma x}: p \in \omega\right\} \circ \bigcup\left\{{ }_{q} S_{\sigma x}: q \in \omega\right\}\right)[x] \\
& \left.=\bigcup\left\{{ }_{k} S_{\sigma x} \circ{ }_{k} S_{\sigma x}\right)[x]: k \in \omega\right\} \\
& \subseteq \bigcup\left\{{ }_{k} S_{\sigma}[x]: k \in \omega\right\}=S_{\sigma}[x] .
\end{aligned}
$$

The second line above follows from the first since if $\langle x, y\rangle \in{ }_{p} S_{\sigma x}$ and $\langle y, z\rangle \in{ }_{q} S_{\sigma x}$, then $\langle x, z\rangle \in{ }_{k} S_{\sigma x} \circ{ }_{k} S_{\sigma x}$, where $k=\max \{p, q\}$. Consequently $S$ is a sequential function for $X$. By construction $S \| X_{n}={ }_{n} S$ and $S \in \Gamma$. Let $\mathscr{V}$ be the local uniformity for which $\Gamma$ is a gauge. What we have just shown assures $\mathscr{V} \mid X_{n} \supseteq \mathscr{V}_{n}$, and the definition of $\Gamma$ assures $\mathscr{V} \mid X_{n} \subseteq \mathscr{V}_{n}$. Thus $(X, \mathscr{V})$ is a limit of $\left\{\left(X_{n}, \mathscr{V}_{n}\right): n \in \omega\right\}$.

Suppose $\left\{\left(X_{n}, \mathscr{V}_{n}\right): n \in \omega\right\}$ has a limit; let $L$ be the set of all limit local uniformities for $\left\{\left(X_{n}, \mathscr{V}_{n}\right): n \in \omega\right\}$. Then $\bigcup L$ is a subbase for a local uniformity $\mathscr{V}^{*}$ on $X$; $\mathscr{V}^{*}$ is obviously the required strongest limit. Similarly, if $L$ is the set of all limits which have a given topology, then $\bigcup L$ is a subbase for a strongest limit with that topology.

We shall now attempt some answers to the question of when a nested family of strongly complete locally uniform spaces has a strongly complete limit. As in 
[4] we shall make the following definitions: for each $V \in \mathscr{V}$, let $V^{2}=V \circ V$, and by induction, for each $n \in \omega-\{0\}$, let $V^{n+1}=V^{n} \circ V$. A filter $\mathscr{F}$ on a locally uniform space $(X, \mathscr{V})$ is weak Cauchy of degree $n$ iff $\forall V \in \mathscr{V}, \exists F \in \mathscr{F}: F \times F \subseteq V^{n}$. It is weak Cauchy if for some $n \in \omega$ it is weak Cauchy of degree $n$. A strongly complete locally uniform space is one in which every weak Cauchy filter converges. For each $n \in \omega-\{0\}$, the collection $\left\{V^{n}: V \in \mathscr{V}\right\}$ is a base for a local uniformity $\mathscr{V}^{n}$; it has the same relative topology as $\mathscr{V}$ (by Theorem 1.10 of [4]). A space $(Y, \mathscr{V})$ is a strong extension of subspace $(X, \mathscr{U})$ iff $\forall n \in \omega-\{0\}, \exists m \in \omega-\{0\}: \mathscr{U}^{m} \subseteq \mathscr{V}^{n} \mid X$.

2.4 THEOREM. Suppose $\left\{\left(X_{n}, \mathscr{V}_{n}\right): n \in \omega\right\}$ is a nested sequence of strongly complete locally uniform spaces. If $(X, \mathscr{V})$ is a limit of the sequence and is a strong extension of each $\left(X_{n}, \mathscr{V}_{n}\right)$, then the strongest limit which generates the same topology as $(X, \mathscr{V})$ is strongly complete.

Proof. Let $(X, \mathscr{V})$ be a limit which is a strong extension of each $\left(X_{n}, \mathscr{V}_{n}\right)$. Let $\left(X, \mathscr{V}^{*}\right)$ be the strongest limit generating the same topology as $(X, \mathscr{V})$. From the fact that $\mathscr{V}^{*}$ is stronger than $\mathscr{V}$, it follows easily that $\mathscr{V}^{*}$ is also a strong extension of each $\left(X_{n}, \mathscr{V}_{n}\right)$. Notice that if $V$ is any $\mathscr{V}$-neighborhood of the diagonal in $X \times X$ such that $\forall n \in \omega, V \cap\left(X_{n} \times X_{n}\right) \in \mathscr{V}_{n}$, then $\{V\} \cup \mathscr{V}$ is a subbase for a limit local uniformity with the same relative topology as $\mathscr{V}$; consequently, $V \in \mathscr{V}^{*}$ by the maximality of $\mathscr{V}^{*}$. Let $\mathscr{F}$ be a weak Cauchy filter of degree $p$ on $\left(X, \mathscr{V}^{*}\right)$.

First we consider the case for which there is a subspace $X_{n}$ such that $\forall V \in \mathscr{V} *$, $\exists F \in \mathscr{F}: F \times F \subseteq V^{p}$ and $V^{p}[F] \cap X_{n} \neq \varnothing$. Then $\forall F \in \mathscr{F}, \forall V \in \mathscr{V}^{*}, V^{2 p}[F] \cap X_{n}$ $\neq \varnothing$; hence $\mathscr{F}^{\prime}=\left\{V^{2 p}[F]: F \in \mathscr{F}, V \in \mathscr{V}^{*}\right\}$ is a weak Cauchy filter base of degree $5 p$ whose restriction to $X_{n}$ is also a weak Cauchy filter base by Theorem 3.3 of [4]. Consequently $\mathscr{F}^{\prime} \mid X_{n}$ converges to some point $x \in X_{n}$ since $\left(X_{n}, \mathscr{V}_{n}\right)$ is strongly complete. But then $\mathscr{F}$ must also converge to $x$.

Now consider the contrary case in which $\forall X_{n}, \exists V \in \mathscr{V}^{*}, \forall F \in \mathscr{F}$; if $F \times F \subseteq V^{p}$, then $V^{p}[F] \cap X_{n}=\varnothing$. We shall derive a contradiction. First we may construct a nested sequence $\left\{V_{k}: k \in \omega\right\}$ of symmetric elements of $\mathscr{V}^{*}$ such that $\forall X_{k}, \forall F \in \mathscr{F}$; if $F \times F \subseteq V_{k}^{p}$, then $V_{k}^{p}[F] \cap X_{k}=\varnothing$. Next, let $P=\bigcup\left\{\left(V_{k}\left[X_{k}\right] \times V_{k}\left[X_{k}\right]\right) \cap V_{k}: k \in \omega\right\}$. $P$ is a neighborhood of the diagonal in $X \times X$, and $\forall n \in \omega, \quad V_{n} \cap\left(X_{n} \times X_{n}\right)$ $\subseteq P \cap\left(X_{n} \times X_{n}\right) \in \mathscr{V}_{n}$; hence $P \in \mathscr{V}^{*}$. Since $\mathscr{F}$ is weak Cauchy of degree $p$, we may pick $F \in \mathscr{F}$ so that $F \times F \subseteq P^{p}$. We shall show by induction that $\forall n \in \omega, F \cap V_{n}^{p}\left[X_{n}\right]$ $=\varnothing$. First, $F \cap V_{0}^{p}\left[X_{0}\right]=\varnothing: P \subseteq V_{0}$ since the $V_{k}$ 's are nested; $F \times F \subseteq P^{p} \subseteq V_{0}^{p}$; so $V_{0}^{p}[F] \cap X_{0}=\varnothing$, and thus $F \cap V_{0}^{p}\left[X_{0}\right]=\varnothing$ since $V_{0}$ is symmetric. Next assume, given $n>0$, that $\forall m<n, F \cap V_{m}^{p}\left[X_{m}\right]=\varnothing$. Since $\left\{V_{k}: k \in \omega\right\}$ is nested, we have that

$$
\begin{aligned}
P^{p} & \subseteq \bigcup\left\{\left(V_{k}^{p}\left[X_{k}\right] \times V_{k}^{p}\left[X_{k}\right]\right) \cap V_{k}^{p}: k \in \omega\right\} \\
& \subseteq V_{n}^{p} \cup \bigcup_{k=0}^{n-1}\left(V_{k}^{p}\left[X_{k}\right] \times V_{k}^{p}\left[X_{k}\right]\right) .
\end{aligned}
$$


Since $F \times F \subseteq P^{p}$ and $\forall k<n,(F \times F) \cap\left(V_{k}^{p}\left[X_{k}\right] \times V_{k}^{p}\left[X_{k}\right]\right)=\varnothing$, it must be that $F \times F \subseteq V_{n}^{p}$; but then $F \cap V_{n}^{p}\left[X_{n}\right]=\varnothing$, by choice of $V_{n}$. Hence

$$
F \cap \bigcup\left\{V_{k}^{p}\left[X_{k}\right]: k \in \omega\right\}=\varnothing,
$$

contradicting the fact that $\mathscr{F}$ is a filter on $X$.

The above theorem is less than ideal since what would be most useful to have is a condition on the spaces $\left(X_{n}, \mathscr{V}_{n}\right)$ which would guarantee the existence of a strongly complete limit, without assuming the existence of a limit which is a strong extension. This latter condition cannot, however, be dropped, as is shown by the following example.

2.5 Example of a nested sequence $\left\{\left(X_{n}, \mathscr{V}_{n}\right): n \in \omega\right\}$ of strongly complete locally uniform spaces which has a limit but not a strongly complete limit. Moreover, for $m<n \in \omega,\left(X_{n}, \mathscr{V}_{n}\right)$ is a strong extension of $\left(X_{m}, \mathscr{V}_{m}\right)$.

For each $n \in \omega$, let $X_{n}=\boldsymbol{R} \times \omega \times(n+2)$. We define a structure on each $X_{n}$ as follows: for each $p \in \omega-\{0\}$, we define $V_{n p}$ by requiring that $\forall x, y \in X_{n},\langle x, y\rangle$ $\in V_{n p}$ iff

(i) $\left|x_{1}-y_{1}\right|<1 / p, x_{2}=y_{2}$, and $x_{3}=y_{3}$; or

(ii) $x_{1}, y_{1}>p, x_{2}=y_{2}>p$, and $x_{3}=y_{3}$; or

(iii) $x_{1}=y_{1}>p, x_{2}=y_{2}$, and $x_{3} y_{3}=0$; or

(iv) $x_{1}=y_{1}>p$ and $\left(\left(x_{3}=0\right.\right.$ and $\left.x_{2}=y_{2}+y_{3}\right)$ or $y_{3}=0$ and $\left.y_{2}=x_{2}+x_{3}\right)$.

It is not difficult to check that $\left\{V_{n p}: p \in \omega\right\}$ is a subbase for a local uniformity $\mathscr{V}_{n}$. To show that each space $\left(X_{n}, \mathscr{V}_{n}\right)$ is strongly complete, one may argue as follows: Suppose $\mathscr{F}$ is a weak Cauchy filter of degree $k$ on $\left(X_{n}, \mathscr{V}_{n}\right)$. First notice that $\max \left\{\left|u_{2}-v_{2}\right|:\langle u, v\rangle \in V_{n p}\right\}=n+1$. So if $F \in \mathscr{F}$ and $\langle x, y\rangle \in F \times F \subseteq V_{n p}^{k}$, then $\left|x_{2}-y_{2}\right| \leqq k(n+1)$. Hence $\mathscr{F}$ must restrict to some subspace of the form $\boldsymbol{R} \times m \times(n+2)$. But then $\mathscr{F}$ must converge in one of these $m(n+2)$ copies of $\boldsymbol{R}$.

Let $X_{\omega}=\bigcup\left\{X_{j}: j \in \omega\right\}=\boldsymbol{R} \times \omega \times \omega$. For each sequence $\alpha$ of integers, let $V_{\alpha}$ $=\bigcup\left\{V_{j \alpha_{j}}: j \in \omega\right\}$. It is a straightforward task to check that the $V_{\alpha}$ 's form a subbase for a local uniformity $\mathscr{V}_{\omega} .\left(X_{\omega}, \mathscr{V}_{\omega}\right)$ is clearly the strongest limit for $\left\{\left(X_{n}, \mathscr{V}_{n}\right): n \in \omega\right\}$. To show that $\left\{\left(X_{n}, \mathscr{V}_{n}\right): n \in \omega\right\}$ does not have a strongly complete limit, it suffices to show $\left(X_{\omega}, \mathscr{V}_{\omega}\right)$ is not strongly complete. $\forall n \in \omega$, let $F_{n}=\left\{x \in X_{\omega}: x_{1}, x_{2}>n\right.$ and $\left.x_{3}=0\right\}$. Then $\left\{F_{n}: n \in \omega\right\}$ is a base for a nonconvergent filter $\mathscr{F}$ on $X_{\omega}$. Pick $V_{\alpha} \in \mathscr{V}_{\omega}$. Pick $x, y \in F_{\alpha_{0}}$, let $a=\left|x_{2}-y_{2}\right|$, then from the definition of the $V_{n p}$ 's, one can verify that $F_{\alpha_{0}} \times F_{\alpha_{0}} \subseteq V_{0 \alpha_{0}} \circ V_{a \alpha_{a}} \circ V_{a \alpha_{a}} \circ V_{0 \alpha_{0}} \subseteq V_{\alpha}^{4}$. Hence $\mathscr{F}$ is weak Cauchy of degree 4.

Finally, if $m<n \in \omega$, then $\left(X_{n}, \mathscr{V}_{n}\right)$ is a strong extension of $\left(X_{m}, \mathscr{V}_{m}\right)$ : For each $p, k \in \omega-\{0\}$, one can, after some calculation, estimate that $V_{n p}^{k} \cap\left(X_{m} \times X_{m}\right)$ $\subseteq V_{m p}^{4 k(2 n+2)}$. Consequently, $\mathscr{V}_{m}^{4 k(2 n+2)} \subseteq \mathscr{V}_{n}^{k} \mid X_{n}$.

\section{Hewitt spaces.}

Definition. A Hewitt space is a nontrivial regular topological space on which every real-valued continuous function is constant. 
First we will consider a general method for constructing Hewitt spaces, and then specialize to show what types of spaces may be produced by the construction. Though the procedure used in Hewitt's example is different from what we will use in the following construction, both procedures use the following ideas: As in [4], we shall say that a bridge space is a regular topological space $B$ together with a pair of points $a^{-}$and $a^{+}$in $B$ which have disjoint closures, but which are functionally linked in the sense that for any real-valued continuous function $f$ on $B, f\left(a^{-}\right)$ $=f\left(a^{+}\right)$. To show that a regular space $H$ is a Hewitt space, it would suffice to show for some bridge space $B$ with $a^{-}$and $a^{+}$that for every two points $x \neq y \in H, H$ contained a copy of $B$ with $a^{-}$and $a^{+}$represented by $x$ and $y$.

3.1 Lemma. Suppose $(X, \mathscr{V})$ is a locally uniform space and $Y$ is a closed subset of $X$ such that $\forall V \in \mathscr{V}, \exists U \in \mathscr{V}: U^{2}[Y] \subseteq V[Y]$. Then

$$
\{V \cup(V[Y] \times Y) \cup(Y \times V[Y]): V \in \mathscr{V}\}
$$

is a base for a local uniformity $\mathscr{V} / Y$. The points of $Y$ are identified in the $\mathscr{V} / Y$ topology.

Proof. For each $V \in \mathscr{V}$, let $V_{Y}=V \cup(V[Y] \times Y) \cup(Y \times V[Y])$. It is clear that $\left\{V_{Y}: V \in \mathscr{V}\right\}$ is a filter base. If $V$ is symmetric, then so is $V_{Y}$. Each $V_{Y}$ contains the diagonal in $X \times X$. Given $x \in X$ and $V \in \mathscr{V}$, if $x \in X-Y$, then since $Y$ is closed, we may choose $U \in \mathscr{V}$ so that $(U \circ U)[x] \subseteq V[x]$ and $U[x] \cap U[Y]=\varnothing$; in this case we will have $\left(U_{Y} \circ U_{Y}\right)[x]=(U \circ U)[x] \subseteq V[x]$. If $x \in Y$, we may choose $U \in \mathscr{V}$ so that $(U \circ U)[Y] \subseteq V[Y]$; and in this case $\left(U_{Y} \circ U_{Y}\right)[x]=(U \circ U)[Y] \subseteq V[Y]$ $=V_{Y}[x]$. This shows that $\left\{V_{Y}: V \in \mathscr{V}\right\}$ is a base for a local uniformity. The second statement that the points of $Y$ are identified in the topology of $\mathscr{V} / Y$ is obvious.

Added in proof. Recently it has come to my attention that $\mathrm{H}$. Herrlich has given a topological construction similar to the following one in his paper Wann sind alle stetigen Abbildungen in Y konstant ?, Math. Z. 90 (1965), 152-154.

3.2 LEMma. By means of the following construction, one may use any bridge space $B$ to embed any locally uniform space $(X, \mathscr{U})$ as a closed subspace of a locally uniform space $(H, \mathscr{W})$ which is topologically a Hewitt space.

Proof. First we shall do a preliminary construction, using $B$ to embed $(X, \mathscr{U})$ in a space $(\hat{X}, \hat{\mathscr{U}})$ in such a way that every real-valued continuous function on $\hat{X}$ is constant on $X$. The main construction will then be completed by iterating the preliminary construction, making for each $n \in \omega,\left(X_{n+1}, \mathscr{U}_{n+1}\right) \cong\left(\hat{X}_{n}, \hat{\mathscr{U}}_{n}\right)$. Finally we shall use Theorem 2.3 to choose a limit $(H, \mathscr{W})$ for $\left\{\left(X_{n}, \mathscr{U}_{n}\right): n \in \omega\right\}$. Every real-valued continuous function $f$ on $(H, \mathscr{W})$ will be constant since each $x, y \in H$ will belong to some $X_{n}$, and $f \mid X_{n+1}$ is constant on $X_{n}$.

So for the preliminary construction, we begin with a local uniform space $(X, \mathscr{U})$ and a bridge space $B$ with $a^{-}$and $a^{+}$. Assume, by making identifications if necessary, that $\left\{a^{-}\right\}$and $\left\{a^{+}\right\}$are closed. Let $\mathscr{V}$ be any local uniformity which generates 
the topology of $B$. Let $\hat{X}=X \times B$. Let $\mathscr{U} \times \mathscr{V}$ be the product local uniformity on $\hat{X}$. $\mathscr{U} \times \mathscr{V}$ has a base of sets $U(\times) V=\{\langle x, z ; y, w\rangle:\langle x, y\rangle \in U,\langle z, w\rangle \in V\}$, for $U \in \mathscr{U}$ and $V \in \mathscr{V}$. The set $X \times\left\{a^{+}\right\}$is closed in $\hat{X}$, and we may let

$$
\hat{\mathscr{U}}=(\mathscr{U} \times \mathscr{V}) /\left(X \times\left\{a^{+}\right\}\right)
$$

be the local uniformity given in the above lemma, since for each element $U(\times) V$ of $\mathscr{U} \times \mathscr{V}$, if we choose $W \in \mathscr{V}$ so that $(W \circ W)\left[a^{+}\right] \subseteq V\left[a^{+}\right]$, then

$$
\begin{aligned}
(U(\times) W \circ U(\times) W)\left[X \times\left\{a^{+}\right\}\right] & =X \times(W \circ W)\left[a^{+}\right] \\
& \subseteq X \times V\left[a^{+}\right]=(U(\times) V)\left[X \times\left\{a^{+}\right\}\right] .
\end{aligned}
$$

$\hat{\mathscr{U}}$ has a base of sets of the form

$$
W(U, V)=U(\times) V \cup(X \times X)(\times)\left(V\left[a^{+}\right] \times\left\{a^{+}\right\} \cup\left\{a^{+}\right\} \times V\left[a^{+}\right]\right) .
$$

It is easy to see that the map $x \mapsto\left\langle x, a^{-}\right\rangle$from $(X, \mathscr{U})$ into $(\hat{X}, \hat{\mathscr{U}})$ is an embedding, and we may identify $X$ with the closed subset $X \times\left\{a^{-}\right\}$. To show that any continuous function on $(\hat{X}, \hat{\mathscr{U}})$ is constant on $X$, we first notice that for each given $x \in X$, the map $b \mapsto\langle x, b\rangle$ is an embedding of $(B, \mathscr{V})$ in $(\hat{X}, \hat{\mathscr{U}})$. This is the case since for each $U \in \mathscr{U}$ and each symmetric $V \in \mathscr{V}$,

$$
\begin{aligned}
W(U, V) \cap & (\{x\} \times B) \times(\{x\} \times B) \\
& =\{\langle x, x\rangle\}(\times) V \cup\{\langle x, x\rangle\}(\times)\left(V\left[a^{+}\right] \times\left\{a^{+}\right\} \cup\left\{a^{+}\right\} \times V\left[a^{+}\right]\right) \\
& =\{\langle x, x\rangle\}(\times) V .
\end{aligned}
$$

If $f$ is any continuous function on $\hat{X}$ and $x, y \in X$, then $\{x\} \times B$ and $\{y\} \times B$ are bridge subspaces of $\hat{X}$, so $f\left(\left\langle x, a^{-}\right\rangle\right)=f\left(\left\langle x, a^{+}\right\rangle\right)$and $f\left(\left\langle y, a^{-}\right\rangle\right)=f\left(\left\langle y, a^{+}\right\rangle\right)$. But the points $\left\langle x, a^{+}\right\rangle$and $\left\langle y, a^{+}\right\rangle$are topologically identified, so $f\left(\left\langle x, a^{+}\right\rangle\right)$ $=f\left(\left\langle y, a^{+}\right\rangle\right)$. Putting these things together, we have $f\left(\left\langle x, a^{-}\right\rangle\right)=f\left(\left\langle y, a^{-}\right\rangle\right)$. Therefore $f$ is constant on $X \times\left\{a^{-}\right\}$, the image of $X$.

We next show that any gauge for $(X, \mathscr{U})$ may be extended to a gauge for $(\hat{X}, \hat{\mathscr{U}})$ : Let $\Gamma$ be any gauge for $(X, \mathscr{U})$. Pick $S \in \Gamma$. Let $\mathscr{B}$ be the set of all symmetric $V \in \mathscr{V}$ such that $V\left[a^{-}\right] \cap V\left[a^{+}\right]=\varnothing$. Pick $V_{0} \in \mathscr{B}$; by induction for each $n \in \omega$, choose $V_{n+1} \in \mathscr{B}$ so that $\left(V_{n+1} \circ V_{n+1}\right)\left[a^{-}\right] \subseteq V_{n}\left[a^{-}\right]$. For each $n$-element sequence $\sigma \in{ }^{\omega>} X$, let $\hat{S}_{\sigma}=W\left(S_{\sigma}, V_{n}\right)$. Then for each $x \in X$, our choices guarantee that

$$
\begin{aligned}
\left(\hat{S}_{\sigma x} \circ \hat{S}_{\sigma x}\right)\left[\left\langle x, a^{-}\right\rangle\right] & =\left(S_{\sigma x} \circ S_{\sigma x}\right)[x] \times\left(V_{n+1} \circ V_{n+1}\right)\left[a^{-}\right] \\
& \subseteq S_{\sigma}[x] \times V_{n}\left[a^{-}\right]=\hat{S}_{\sigma}\left[\left\langle x, a^{-}\right\rangle\right] .
\end{aligned}
$$

The bijection $x \mapsto\left\langle x, a^{-}\right\rangle$from $X$ to $X \times\left\{a^{-}\right\}$induces a bijection $\sigma \mapsto \sigma^{*}$ from ${ }^{\omega>} X$ to ${ }^{\omega>}\left(X \times\left\{a^{-}\right\}\right)$. The composite function $\sigma^{*} \mapsto \sigma \mapsto \hat{S}_{\sigma}$ from ${ }^{\omega>}\left(X \times\left\{a^{-}\right\}\right)$to $\hat{\mathscr{U}}$ may now be extended to a sequential function $\hat{S}$ from ${ }^{\omega>} \hat{X}$ to $\hat{\mathscr{U}}$ by induction. Having chosen $\hat{S}_{\tau}$ with $\tau \in{ }^{\omega>} \hat{X}$, we just choose for each $x \in \hat{X}$, a symmetric $\hat{S}_{\tau x} \in \hat{\mathscr{U}}$ so that $\left(\hat{S}_{\tau x} \circ \hat{S}_{\tau x}\right)[x] \subseteq \hat{S}_{\tau}[x]$. The function $\hat{S}$ is, in effect, an extension of $S$ since for each $\sigma \in{ }^{\omega>} X$,

$$
\hat{S}_{\sigma^{*}} \cap\left(X \times\left\{a^{-}\right\}\right) \times\left(X \times\left\{a^{-}\right\}\right)=S_{\sigma}(\times)\left\{\left\langle a^{-}, a^{-}\right\rangle\right\} .
$$


Finally, as indicated at the beginning of the construction, we define $\left(X_{n}, \mathscr{U}_{n}\right)$ for each $n \in \omega$ as follows: Let $X_{n}=\left\{x \in{ }^{\omega}(X \cup B): x_{0} \in X ; \forall m>0, x_{m} \in B\right.$; and $\left.\forall k>n, x_{k}=a^{-}\right\}$. Let $H=\bigcup\left\{X_{n}: n \in \omega\right\}$. We may transfer the local uniformity $\mathscr{U}$ on $X$ to an isomorphic local uniformity $\mathscr{U}_{0}$ on $X_{0}$ by means of the bijection $x \mapsto x_{0}$ from $X_{0}$ to $X$. For each $n \in \omega$, we can define a bijection $\beta_{n}$ from $\hat{X}_{n}=X_{n} \times B$ to $X_{n+1}$ as follows: $\forall x \in X_{n}, \forall y \in B, \beta_{n}(x, y)(n+1)=y$, and for $i \neq n+1, \beta_{n}(x, y)(i)$ $=x_{i}$. By induction, for each $n \geqq 0$, having defined $\mathscr{U}_{n}$ on $X_{n}$, we use $\beta_{n}$ to transfer $\hat{\mathscr{U}}_{n}$ on $\hat{X}_{n}$ to an isomorphic local uniformity $\mathscr{U}_{n+1}$ on $X_{n+1}$. For each $n, \beta_{n}$ transfers the embedding $X_{n} \rightarrow X_{n} \times\left\{a^{-}\right\} \subseteq \hat{X}_{n}$ to the identity embedding $X_{n} \rightarrow X_{n} \subseteq X_{n+1}$. Consequently, each gauge on $\left(X_{n}, \mathscr{U}_{n}\right)$ extends to a gauge on $\left(X_{n+1}, \mathscr{U}_{n+1}\right)$. Hence by Theorem 2.3 , we may choose a limit $(H, \mathscr{W})$ for $\left\{\left(X_{n}, \mathscr{U}_{n}\right): n \in \omega\right\}$. This completes the construction.

3.3 Theorem. Suppose $(X, \mathscr{U})$ is any locally uniform space with some of the following properties:

(1) it has cardinality $\boldsymbol{\aleph} \geqq \boldsymbol{\aleph}_{1}$,

(2) its relative topology is separable,

(3) its relative topology is first countable,

(4) it is strongly complete.

Then $(X, \mathscr{U})$ may be embedded as a closed subspace in a locally uniform space which has the same properties, but is topologically a Hewitt space.

Proof. By Construction 5.6 of [4], we may let $(D, \mathscr{V})$ be a locally uniform space which has cardinality $\aleph_{1}$, satisfies conditions (2)-(4), and is topologically a bridge space. Our proof will consist of applying the above construction to $(X, \mathscr{U})$, using $(B, \mathscr{V})$ for the bridge space.

If $(X, \mathscr{U})$ has cardinality $\boldsymbol{\aleph} \geqq \boldsymbol{\aleph}_{1}$, then so does $\hat{X}=X \times B$, and by induction, so does each $X_{n}$. Consequently so will their union $H$. Thus property (1) is preserved.

If $(X, \mathscr{U})$ has a separable topology, then the product $(X, \mathscr{U}) \times(B, \mathscr{V})$ has a separable topology. The topology of $(\hat{X}, \hat{\mathscr{U}})$ is weaker than that of $\mathscr{U} \times \mathscr{V}$, and hence is also separable. By induction each $\left(X_{n}, \mathscr{U}_{n}\right)$ has a separable topology. Consequently, any limit $(H, \mathscr{W})$ being the countable union of separable subspaces, also has a separable topology. Thus property (2) is preserved.

In order to preserve properties (3) and (4), we must be careful about the limit taken at the end of the construction. If the limit $(H, \mathscr{W})$ is too strong, it may not have a first countable topology, and if it is too weak, it may not be strongly complete. So first we shall define a limit $\mathscr{W}$ which has a first countable topology (provided $(X, \mathscr{U})$ does), and then pick the strongest limit $\mathscr{W}^{*}$ which generates the same topology as $\mathscr{W} . \mathscr{W}^{*}$ will be strongly complete provided $\mathscr{U}$ is.

For each $U \in \mathscr{U}$ and $V \in \mathscr{V}$, let

$$
\begin{aligned}
T(U, V)=\{\langle x, y\rangle \in H: & \exists m \in \omega, \forall n>m,\left\langle x_{n}, y_{n}\right\rangle \in V \text { and } \\
& \left.\left(x_{m+1}=a^{+} \text {or } y_{m+1}=a^{+}, \text {or } m=0 \text { and }\left\langle x_{0}, y_{0}\right\rangle \in U\right)\right\} .
\end{aligned}
$$


For each $k \in \omega$, let $T_{k}(U, V)=\left(X_{k} \times X_{k}\right) \cap T(U, V)$. Let $\mathscr{B}=\{T(U, V): U \in \mathscr{U}$, $V \in \mathscr{V}\}$; for each $k \in \omega$, let $\mathscr{B}_{k}=\left\{T_{k}(U, V): U \in \mathscr{U}, V \in \mathscr{V}\right\}$. We want to show that each $\mathscr{B}_{k}$ is a base for $\mathscr{U}_{k}$, and that $\mathscr{B}$ is a base for a limit local uniformity. If $U, U^{\prime} \in \mathscr{U}$ and $V, V^{\prime} \in \mathscr{V}$, then $T\left(U \cap U^{\prime}, V \cap V^{\prime}\right) \subseteq T(U, V) \cap T\left(U^{\prime}, V^{\prime}\right)$, so that $\mathscr{B}$ is a filter base. Consequently so is each $\mathscr{B}_{k}$. We have first $\mathscr{B}_{0}=\mathscr{U}_{0}$; by induction, if $\mathscr{B}_{n}$ is a base for $\mathscr{U}_{n}$, then it is easy to see that $\hat{\mathscr{B}}_{n}=\left\{W\left(T_{n}(U, V), V\right)\right.$ : $U \in \mathscr{U}, V \in \mathscr{V}\}$ is a base for $\hat{\mathscr{U}}_{n}$. Under the isomorphism

$$
\beta_{n}:\left(\hat{X}_{n}, \hat{\mathscr{U}}_{n}\right) \rightarrow\left(X_{n+1}, \mathscr{U}_{n+1}\right) \text {, }
$$

each $W\left(T_{n}(U, V), V\right)$ transfers to $T_{n+1}(U, V)$. Hence $\mathscr{B}_{n+1}$ is a base for $\mathscr{U}_{n+1}$. To show that $\mathscr{B}$ is a base for a local uniformity on $H$, we notice first that each $T(U, V)$ contains the diagonal in $H \times H$, and if $U$ and $V$ are symmetric, then so is $T(U, V)$. Next, given $T(U, V) \in \mathscr{B}$ and $x \in H$, let $n$ be the least integer such that $x \in X_{n}$; then we may choose $U^{\prime} \in \mathscr{U}$ and $V^{\prime} \in \mathscr{V}$ so that $T_{n}\left(U^{\prime}, V^{\prime}\right)^{2}[x] \subseteq T_{n}(U, V)[x]$, and so that $V^{\prime 2}\left[a^{-}\right] \subseteq V\left[a^{-}\right]$and $V^{\prime}\left[a^{+}\right] \cap V^{\prime}\left[a^{-}\right]=\varnothing$. Using induction we assume for $m \geqq n$ that $T_{m}\left(U^{\prime}, V^{\prime}\right)^{2}[x] \subseteq T_{m}(U, V)[x]$; then

$$
\begin{aligned}
W\left(T_{m}\left(U^{\prime}, V^{\prime}\right), V^{\prime}\right)^{2}\left[\left\langle x, a^{-}\right\rangle\right] & =T_{m}\left(U^{\prime}, V^{\prime}\right)^{2}[x] \times V^{2}\left[a^{-}\right] \\
& \subseteq T_{m}(U, V)[x] \times V\left[a^{-}\right] \\
& \subseteq W\left(T_{m}(U, V)\left[\left\langle x, a^{-}\right\rangle\right] .\right.
\end{aligned}
$$

Transferring this calculation from $\hat{X}_{m}$ to $X_{m+1}$, we have $T_{m+1}\left(U^{\prime}, V^{\prime}\right)^{2}[x]$ $\subseteq T_{m+1}(U, V)[x]$. Taking the union over all subspaces, we have $T\left(U^{\prime}, V^{\prime}\right)^{2}[x]$ $\subseteq T(U, V)[x]$. Hence $\mathscr{B}$ is a base for a local uniformity $\mathscr{W}$ on $H$. Since $\forall n \in \omega$, $\mathscr{B} \mid X_{n}=\mathscr{B}_{n},(H, \mathscr{W})$ is a limit of the sequence $\left\{\left(X_{n}, \mathscr{V}_{n}\right): n \in \omega\right\}$.

Now we suppose $(X, \mathscr{U})$ has a first countable topology, and show that $(H, \mathscr{W})$ does. Pick $x \in H$; let $n$ be the least number for which $x \in X_{n}$. Let $\left\{U_{k}\left[x_{0}\right]: k \in \omega\right\}$ be a countable base for the neighborhood system at $x_{0} \in X$, with each $U_{k} \in \mathscr{U}$. For each $m$ with $0<m \leqq n+1$, let $\left\{V_{m k}\left[x_{m}\right]: k \in \omega\right\}$ be a countable base for the neighborhood system at $x_{m} \in B$, with each $V_{m k} \in \mathscr{V}$. For each $k \in \omega$, let $V_{k}$ $=\bigcap\left\{V_{m k}: 0<m \leqq n+1\right\}$. We now show that $\left\{T\left(U_{k}, V_{k}\right)[x]: k \in \omega\right\}$ is a countable base for the $\mathscr{W}$-neighborhood system at $x$ : Given $U \in \mathscr{U}$ and $V \in \mathscr{V}$, suppose that $U_{k}\left[x_{0}\right] \subseteq U\left[x_{0}\right]$, and for $0<m \leqq n+1$, that $V_{k}\left[x_{m}\right] \subseteq V\left[x_{m}\right]$; then it is easy to see that $T\left(U_{k}, V_{k}\right)[x] \subseteq T(U, V)[x]$. Hence $(H, \mathscr{W})$ has a first countable topology. Thus property (3) is preserved.

Finally, suppose $(X, \mathscr{U})$ is strongly complete. We shall need the following facts:

(1) A closed subspace of a strongly complete locally uniform space is strongly complete.

(2) The product of strongly complete locally uniform spaces is strongly complete. These statements are essentially part of Theorems 6.14 and 6.15 of [4]. (1) is true since a filter which is weak Cauchy on a subspace must be weak Cauchy on the whole space. (2) follows directly from the fact that for any product of locally uniform spaces, the coordinate projections preserve weak Cauchy filters. 
First we show by induction that each $\left(X_{n}, \mathscr{U}_{n}\right)$ is strongly complete. $\left(X_{0}, \mathscr{U}_{0}\right)$ is; for $n \geqq 0$ suppose that $\left(X_{n}, \mathscr{U}_{n}\right)$ is. Let $\mathscr{F}$ be a weak Cauchy filter of degree $p$ on $\left(\hat{X}_{n}, \hat{\mathscr{U}}_{n}\right)$. If each $F \in \mathscr{F}$ meets each neighborhood of $X_{n} \times\left\{a^{+}\right\}$, then $\mathscr{F}$ converges to the identified points of $X_{n} \times\left\{a^{+}\right\}$. On the other hand, suppose there is a neighborhood $N$ of $a^{+}$such that $\mathscr{F}$ restricts to $X_{n} \times(B-N)$. Pick $U \in \mathscr{U}$; pick $V \in \mathscr{V}$ so that $V^{p+1}\left[a^{+}\right] \subseteq N$; let $M=\left(X-V\left[a^{+}\right]\right)^{-}$. Then if we choose $F \in \mathscr{F} \mid X_{n} \times(B-N)$ so that $F \times F \subseteq W\left(T_{n}(U, V), V\right)^{p}$, we have

$$
\begin{aligned}
F \times F & \subseteq W\left(T_{n}(U, V), V\right)^{p} \cap\left(X_{n} \times(B-N)\right) \times\left(X_{n} \times(B-N)\right) \\
& \subseteq\left[W\left(T_{n}(U, V), V\right) \cap\left(X_{n} \times M\right) \times\left(X_{n} \times M\right)\right]^{p} .
\end{aligned}
$$

Hence $\mathscr{F} \mid X_{n} \times(B-N)$ is weak Cauchy of degree $p$ in $X_{n} \times M .(M, \mathscr{V} \mid M)$ is a closed, and therefore strongly complete, subspace of $(B, \mathscr{V})$. The space $\left(X_{n} \times M, \hat{\mathscr{U}}_{n} \mid X_{n} \times M\right)$ is equal to $\left(X_{n} \times M, \mathscr{U}_{n} \times \mathscr{V} \mid M\right)$ by construction, and being the product of strongly complete spaces, is strongly complete. Hence $\mathscr{F} \mid X_{n}$ $\times(B-N)$ converges in $X_{n} \times M$; so $\mathscr{F}$ does too. Therefore $\left(\hat{X}_{n}, \hat{\mathscr{U}}_{n}\right)$, and thus $\left(X_{n+1}, \mathscr{U}_{n+1}\right)$ is strongly complete.

Next, we can show that $(H, \mathscr{W})$ is a strong extension of each $\left(X_{n}, \mathscr{U}_{n}\right)$ as follows: Pick $k \in \omega$; if $U \in \mathscr{U}$, and $V \in \mathscr{V}$ is such that $\left\{\left\langle a^{-}, a^{+}\right\rangle,\left\langle a^{+}, a^{-}\right\rangle\right\} \cap V^{k}=\varnothing$, then it is easy to see that $\forall m \geqq n$,

$$
T_{m+1}(U, V)^{k} \cap\left(X_{m} \times X_{m}\right)=T_{m}(U, V)^{k} ;
$$

thus using induction, $\forall m \geqq n$,

$$
T_{m}(U, V)^{k} \cap\left(X_{n} \times X_{n}\right)=T_{n}(U, V)^{k} ;
$$

thus

$$
T(U, V)^{k} \cap\left(X_{n} \times X_{n}\right)=T_{n}(U, V)^{k} .
$$

Hence $(H, \mathscr{W})$ is a strong extension of $\left(X_{n}, \mathscr{U}_{n}\right)$.

Finally, if $\mathscr{W}^{*}$ is the strongest limit which generates the same topology as $\mathscr{W}$, then by Theorem $2.4,\left(H, \mathscr{W}^{*}\right)$ is strongly complete. Thus property (4) is preserved.

ACKnowledgements. I wish to thank T. P. Srinivasan and E. Akin for their conversations and suggestions concerning this work.

\section{REFERENCES}

1. E. Hewitt, On two problems of Urysohn, Ann. of Math. (2) 47 (1946), 503-509. MR 8, 165.

2. Josef Novak, Regular space on which every continuous function is constant, Casopis. Pěst. Mat. Fys. 73 (1948), 58-68. MR 10, 467.

3. J. R. Isbell, On finite dimensional uniform spaces, Pacific J. Math. 9 (1959), 107-121. MR 21 \#4407.

4. James Williams, Locally uniform spaces, Trans. Amer. Math. Soc. 168 (1972), 435-469.

Department of Mathematics, Bowling Green State University, Bowling Green; Онго 43403 